\title{
Extracting dynamics from threshold-crossing interspike intervals: Possibilities and
} limitations

\author{
Pavlov, A. N.; Sosnovtseva, O. V.; Mosekilde, Erik; Anishchenko, V. S.
}

Published in:

Physical Review E. Statistical, Nonlinear, and Soft Matter Physics

Link to article, DOI:

10.1103/PhysRevE.61.5033

Publication date:

2000

Document Version

Publisher's PDF, also known as Version of record

Link back to DTU Orbit

Citation (APA):

Pavlov, A. N., Sosnovtseva, O. V., Mosekilde, E., \& Anishchenko, V. S. (2000). Extracting dynamics from threshold-crossing interspike intervals: Possibilities and limitations. Physical Review E. Statistical, Nonlinear, and Soft Matter Physics, 61(5), 5033-5044. https://doi.org/10.1103/PhysRevE.61.5033

\section{General rights}

Copyright and moral rights for the publications made accessible in the public portal are retained by the authors and/or other copyright owners and it is a condition of accessing publications that users recognise and abide by the legal requirements associated with these rights.

- Users may download and print one copy of any publication from the public portal for the purpose of private study or research.

- You may not further distribute the material or use it for any profit-making activity or commercial gain

- You may freely distribute the URL identifying the publication in the public portal 


\title{
Extracting dynamics from threshold-crossing interspike intervals: Possibilities and limitations
}

\author{
Alexey N. Pavlov, ${ }^{1}$ Olga V. Sosnovtseva, ${ }^{1}$ Erik Mosekilde, ${ }^{2}$ and Vadim S. Anishchenko \\ ${ }^{1}$ Nonlinear Dynamics Laboratory, Department of Physics, Saratov State University, Astrakhanskaya Street 83, 410026, Saratov, Russia \\ ${ }^{2}$ Department of Physics, Technical University of Denmark, 2800 Lyngby, Denmark
}

(Received 26 July 1999; revised manuscript received 16 December 1999)

\begin{abstract}
In this paper we estimate dynamical characteristics of chaotic attractors from sequences of thresholdcrossing interspike intervals, and study how the choice of the threshold level (which sets the equation of a secant plane) influences the results of the numerical computations. Under quite general conditions we show that the largest Lyapunov exponent can be estimated from a series of return times to the secant plane, even in the case when some of the loops of the phase space trajectory fail to cross this plane.
\end{abstract}

PACS number(s): 05.45.- a

\section{INTRODUCTION}

The continuous-time evolution of many systems is accompanied by striking changes in the physical variables that are repeated more or less regularly. This situation typically arises in the biological sciences, and is encountered in neurobiology (neuron firings corresponding to voltage spikes $[1]$ ), cardiology ( $R$ peaks of electrocardiograms [2]), membrane biology (bursting oscillations of the cell membrane potential [3]), etc. Systems with this type of dynamics are often analyzed by processing time intervals between the relevant events [for example, interspike intervals (ISI's) [4]].

Different models of spike generation are known. Within the framework of integrate-and-fire (IF) models [5-8], a signal $S(t)$, being a function of the variables of a lowdimensional dynamical system (DS) is integrated from some moment $T_{0}$. The times $T_{i}$ when spikes occur can then be defined by the equation

$$
\int_{T_{i}}^{T_{i+1}} S(t) d t=\theta, \quad I_{i}=T_{i+1}-T_{i}, \quad i=1,2,3, \ldots,
$$

where $\theta$ is a firing threshold, and $I_{i}$ are the interspike intervals (IF ISI's). When the specified threshold $\theta$ is reached, a sharp pulse is generated [Fig. 1(a)], and the value of the integral is reset to zero.

Threshold-crossing models (TC) [6], on the other hand, assume the existence of a threshold level $\Theta$, which defines the equation of a secant plane $S=\Theta[S(t)$ is now a variable of a DS], and measure time intervals between successive crossings of the given level by the signal $S(t)$ in one direction, e.g., from below and up (TC ISI's) [Fig. 1(b)]. From the viewpoint of dynamical system theory, TC ISI's are the times when the phase trajectory returns to the secant plane.

The problem of ISI analysis is important when, for whatever reason, the full signal $S(t)$ cannot be recorded, and only a sequence of firing times is available in the course of the experiment. A sensory neuron that transforms a time-varying input signal $S(t)$ into the resulting output spike trains may serve as a classical example. This transformation was previously investigated within the framework of information theory [9].

A sensory neuron represents a threshold device with an input and an output: at the input a signal of complex structure is received, and at the output a series of pulses is mea- sured. Since the output pulses are identical and their shape does not depend on an external force, the information about the properties of the input signal can be encoded only in time intervals between neuron firings. A question arises: How can a characterization of the input signal be provided when processing a spike train only? During the last years, new insight into the analysis of spike-train data has appeared. An ISI may be considered as a new state variable allowing us to characterize the low-dimensional dynamics at the input of a neuron from the observed spike train [5-8,10-12]. Following Sauer [5], the attractor of a chaotic system can be reconstructed [13] using a sequence of time intervals only, and deterministically driven IF ISI sequences can be distinguished from stochastically driven series on the basis of a calculation of the prediction error. Sauer [5,7] also proved an embedding theorem for IF ISI's. Following Hegger and Kantz [10], this theorem is valid for return times as well. A detailed study of how the different properties of a chaotic forcing are reflected in an output IF ISI series was performed by Racicot and Longtin [8]. Ding and Yang [12] demonstrated the results of chaos control based on TC ISI process-
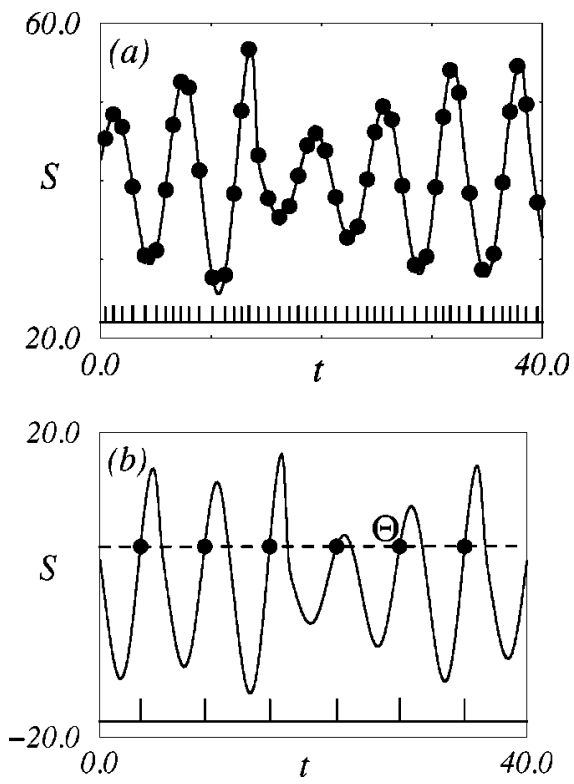

FIG. 1. Models of spike generation: (a) integrate and fire, and (b) threshold crossing. Black points indicate the time moments when a threshold level is reached. A sequence of spikes representing the output signal is given at the bottom of each figure. 
ing. In our previous contribution [14], an interpretation of return times based on an analytical signal approach and the notion of an instantaneous frequency for chaotic oscillations was suggested, and metrical and dynamical characteristics of some attractors were computed from TC ISI's.

In the present work we study how the choice of a firing threshold and the structure of return times influence the results of a reconstruction. Our investigations will be carried out through the estimation of the largest Lyapunov characteristic exponent (LCE) $\lambda_{1}$, which is perhaps the most informative invariant of a complicated dynamical process [15]. We shall discuss the conditions under which the value of $\lambda_{1}$ can be estimated from a sequence of TC ISI's using standard algorithms without modifications.

Our paper is organized as follows. Section II briefly discusses the methods for LCE computation. Features of the reconstruction of dynamical characteristics from spike-train data are the subject of Sec. III. In Sec. IV we focus on the influence of the firing threshold and on a qualitative explanation of the obtained results.

\section{METHODS FOR ESTIMATION OF THE LARGEST LYAPUNOV EXPONENT}

In this section we shall briefly discuss the methods of LCE computation in order to introduce some algorithmic parameters of importance for further analysis. It is well known that an exponential instability of the trajectories is a characteristic of dynamical chaos. A quantitative measure of this instability is the positive LCE characterizing the sensitivity of a DS to variations in the initial conditions. The number of positive exponents in the LCE spectrum is determined by the number of expanding directions of unstable periodic orbits, although more complicated situations may also arise [16]. Within the framework of the present study we shall limit ourselves to chaotic regimes with a single positive Lyapunov exponent $\lambda_{1}$. While computing $\lambda_{1}$ we shall assume that the phase trajectory, being the solution of a DS for the chosen initial conditions, is typical. Otherwise, the value of the exponent estimated during a finite time span $T$ can significantly differ from the value which is obtained theoretically in the limit $T \rightarrow \infty$. As an example, Gambaudo and Tresser [17] described a map with very long transient processes (during up to 1500000 iterations it behaves "chaotically" before falling into the periodic orbit). From the viewpoint of the LCE calculation this means that an apparently stable positive value $\lambda_{1}$ would be attained after part of the transient process, and only after a very long calculation time will the value decrease to zero. Long transient processes and complex variations of the Lyapunov spectrum typically occur in the vicinity of homoclinic trajectories of a saddle cycle under the destruction of quasiperiodic oscillations [18]. The phenomenon of riddled basins of attraction that can be observed, for instance, in connection with chaotic synchronization [19], also depends on the fact that the (transverse) Lyapunov exponent for specific orbits can differ markedly from the corresponding exponent for the typical trajectory [20]. To characterize the behavior of a typical phase trajectory one sometimes uses the concept of finite-time Lyapunov exponents, which quantify the rate of expansion or contraction during a finite-time span $T[16]$.
If we know the equations of a DS generating a phase trajectory, e.g., in the form of a set of ordinary differential equations,

$$
\frac{d \vec{x}}{d t}=\vec{f}(\vec{x}, \vec{\mu}), \quad \vec{x} \in R^{n}, \quad \vec{\mu} \in R^{m},
$$

where $\vec{x}$ is the state vector, $\vec{f}$ is the nonlinear vector function, and $\vec{\mu}$ is the parameter vector, then the maximal Lyapunov exponent (or the full LCE spectrum) can be estimated using the algorithm suggested by Benettin et al. [21] and by Shimada and Nagashima [22]. This technique is referred to as the "standard" algorithm for LCE computing [23]. Detailed discussions of the various aspects of this technique may be found in several publications $[24,25]$.

The problem of estimation of the largest Lyapunov exponent becomes complicated, if Eqs. (2) are unknown. However, at present a large number of methods for LCE computation from experimental data have been developed [26-28]. The various ways in which to obtain dynamical characteristics of chaotic attractors from observed time series are discussed in Refs. [29]. In the present study we use the method suggested by Wolf et al. [26]. This algorithm uses the fact that in many systems the growth of infinitesimal perturbations is exponential,

$$
r(t)=r_{0} e^{\lambda_{1}\left(t_{0}\right)\left(t-t_{0}\right)},
$$

where $r_{0}$ is the distance between the so-called fiducial trajectory and its neighboring orbit at the moment $t_{0}$, and the increment $\lambda_{1}\left(t_{0}\right)$ defines the evolution in time of an initial spatial separation between two state vectors. (The local growth of perturbations is the function of a point in the phase space. To show this circumstance, in this section we indicate the dependence $\lambda_{1}\left(t_{0}\right)$, since the value of the time moment corresponds to some point of the fiducial trajectory.) The average along a typical phase trajectory value of the increment $\lambda_{1}\left(t_{0}\right)$ may be taken as the estimation of the largest LCE. In practice, the method of Ref. [26] allows one to compute the rate of divergence for small, but finite perturbations. This may lead to problems in interpretation of the obtained results from the mathematical viewpoint, although it was analytically shown that the exponential growth may also persist for a finite distance between the orbits [25]. Since dependence (3) is carried out only for small enough values $r(t)$, renormalizations must be performed while computing $\lambda_{1}$. In this procedure, new replacement vectors are chosen in the direction of the most rapid divergence and with specific sizes. Dealing with a single trajectory limits the possibility of the choice of replacement vectors, and we need to search for a compromise between minimizing its size and reducing the orientational error. The restrictions of the vector size can be formulated as follows:

$$
l_{1}<r(t)<l_{2} .
$$

We need to select some minimal value $l_{1}$ such that the effect of noise in the experimental data is not accentuated by the numerical calculations. The value $l_{2}$ sets the condition of a linear approach (or exponential divergence), and can be introduced usually as some fraction of the size of the attractor (e.g., 5-10\%). In the frames of a "variable evolution time" 
algorithm [26], the replacement is performed when the distance between the orbits no longer satisfies the condition of linear approximation $\left(r>l_{2}\right)$. [As an alternative we may use the "fixed evolution time" algorithm [26], with the replacement procedures at regular intervals in time, provided that the distance $r(t)$ does not become too large during the given time spans.] Since the estimation of $\lambda_{1}$ is based on the restoration of the attractor, the result will depend on the quality of this reconstruction [30]. This leads to the appearance of additional parameters of the numerical computations such as the embedding dimension, the time delay between the phase space coordinates of the reconstructed attractor, etc. [13].

To end our brief description of the techniques for computing the largest LCE, let us emphasize a final important circumstance. In our study we consider the case when the signal being processed (an input signal of IF or TC model) is a one-dimensional projection of a phase trajectory which belongs to a chaotic attractor. When dealing with experimental data the dynamical nature of a time series often cannot be established. If the signal being analyzed is not deterministic we cannot speak about Lyapunov exponents. In this case, the algorithm of Ref. [26] defines only some quantitative characteristics of the sensitivity to the choice of initial conditions [31], or a measure of predictability.

\section{COMPUTING THE LARGEST LYAPUNOV EXPONENT FROM INTERSPIKE INTERVALS}

The problem of restoration of the chaotic attractor with the one-dimensional projection acting as a forcing signal of an IF model from interspike intervals was studied in a number of publications $[5-8,11]$. The quality of the reconstruction depends on the choice of a firing threshold $\theta$. As shown by Racicot and Longtin [8], the mean value theorem in some approximation allows us to consider an IF ISI at high firing rates as a nonlinear transformation of an input signal $S(t)$ :

$$
I_{i} \approx \theta / S_{i}, \quad S_{i}=S\left(T_{i}\right) .
$$

Since the largest LCE is invariant under nonlinear transformations, the value of $\lambda_{1}$ calculated from IF ISI's should coincide with the Lyapunov exponent estimated from $S(t)$. However, the problem is to find a sufficiently good technique.

On the one hand, we can consider ISI's only as a discrete sequence $I_{1}, I_{2}, \ldots, I_{N}$, restore the attractor using the delay method $\left(I_{i}, I_{i+1}, \ldots, I_{i+m-1}\right)$ [13], and estimate a measure of chaoticity as the average rate of loss of information about the initial conditions per "iteration." The duration of an iteration can be taken approximately as the average value of the interspike intervals $\bar{I}=(1 / N) \sum_{i=1}^{N} I_{i}$. We have carried out such a calculation using the Rössler system

$$
\begin{gathered}
\frac{d x}{d t}=-(y+z), \quad \frac{d y}{d t}=x+a y, \\
\frac{d z}{d t}=b+z(x-c), \quad a=0.15, \quad b=0.2, \quad c=10.0
\end{gathered}
$$

as an example. In analogy with Racicot and Longtin [8] we have chosen $S(t)=x(t)+40$ as the input signal, and have
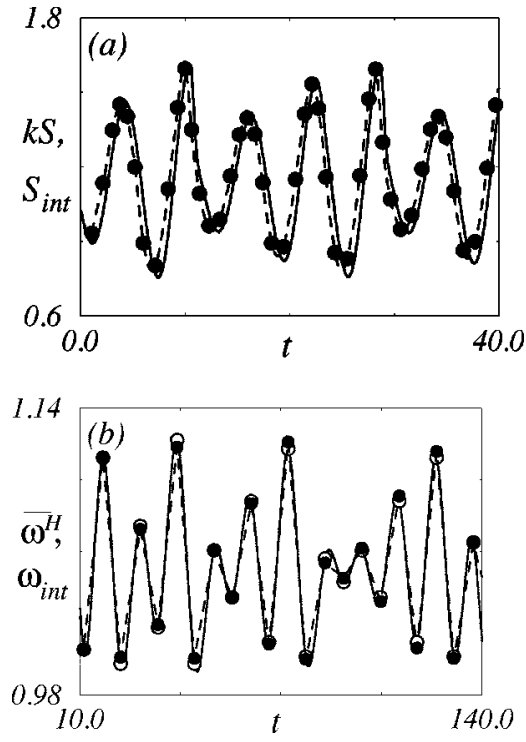

FIG. 2. (a) Solid curve representing the linear transformation of the forcing signal $(1 / \theta) S(t)$, and a dashed curve corresponding to the result of an interpolation of the points $\left(1 / I_{i}\right)\left(T_{i}\right)$. The small shift in time, which has no significance for the calculation of the LCE, can be removed if we take $S_{i}=S\left(\left(T_{i}+T_{i+1}\right) / 2\right)$ in Eq. (5). (b) Black points connected by a dashed line are the points of the average instantaneous frequency obtained via a Hilbert transformation at the time moments appropriate to the crossings of a threshold$\bar{\omega}^{H}\left(T_{i}\right)$; white points connected by solid smooth curve $\omega_{i n t}(t)$ are the values of $\left(2 \pi / I_{i}\right)\left(T_{i}\right)$ at the same time moments.

fixed the threshold $\theta=35$. As a result an underestimated value of the largest Lyapunov exponent was obtained (with an error of about $25 \%$ ).

Another approach to the problem of LCE estimation consists of the following: If the sequence of IF ISI's is known, according to Eq. (5),

$$
\frac{1}{I_{i}} \approx \frac{1}{\theta} S_{i}=k S\left(T_{i}\right)
$$

i.e., at fixed moments $T_{i}$ we can determine the values of the input signal multiplied by some constant $k$. Knowing the values $k S\left(T_{i}\right)$, and aiming to restore the forcing signal as a continuous-time variation, we suggest interpolating a smooth function $S_{\text {int }}(t)$ (e.g., a cubic spline) into the points $1 / I_{i}$ at the moments $T_{i}$. The interpolation will allow us to introduce a constant step in time, and to restore with some accuracy the linear transformation of the input signal, i.e., $S_{\text {int }}(t) \approx k S(t)$ [Fig. 2(a)]. Hence $S_{\text {int }}(t)$ will maintain the metrical and dynamical properties of an attractor corresponding to the chaotic forcing. Clearly, the above procedure is carried out within a certain accuracy, taking into account both the errors of interpolation and the approximate character of Eq. (5). However, as one can see from Fig. 3(a), the value $\lambda_{1}$ computed from the signal $S_{\text {int }}(t)$ using the algorithm of Ref. [26] coincides with the result of an estimation of $\lambda_{1}$ directly from $S(t)$ (i.e., with the largest Lyapunov exponent of the original continuous system). Note that we have obtained a significantly smaller error of calculation than in the previous case (where $\lambda_{1}$ was computed from a discrete sequence of ISI's). We explain this result as follows. An increase of the number of points in the interpolated temporal dependence in com- 

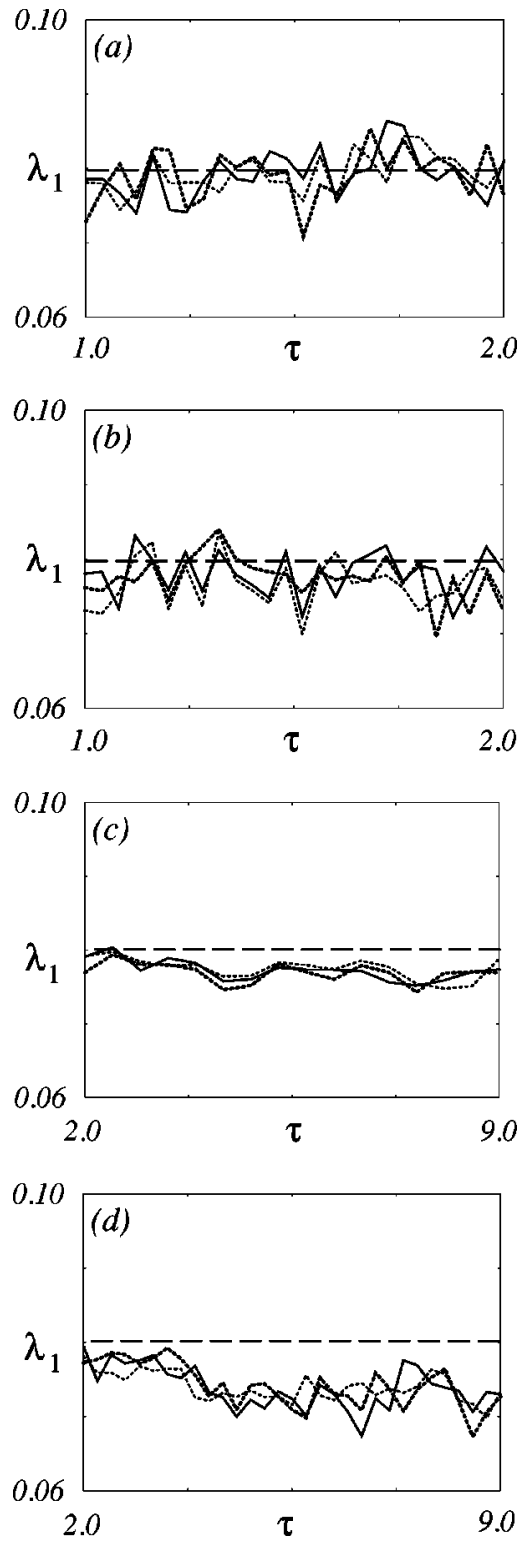

FIG. 3. The largest LCE vs time delay $\tau$ at various values of the parameter $l_{2}$ computed from a sequence of IF ISI's without (a) and with noise (b) and also from a TC ISI series without (c) and with (d) noise. The embedding dimension was chosen to be equal to 5 . Dashed lines indicate the value $\lambda_{1}$ of the original continuous system estimated from the input signal $S(t)$ by means of the algorithm of Ref. [26] $\left(\lambda_{1} \approx 0.083\right)$. The method of Ref. [21] leads to the result $\left(\lambda_{1} \approx 0.09\right)$.

parison with the discrete sequence (i.e., an increase of the number of points in the restored attractor) may lead to a decrease of the orientational error of the replacement vector while computing the LCE using the algorithm of Ref. [26]. To obtain authentic results we have estimated the LCE for different algorithmic parameters such as time delays, embedding dimensions, and parameters $l_{2}$, specifying the condition of the linear approximation [Fig. 3(a)].

The problem of extracting dynamics from a TC ISI series is more complex. A possible approach suggested in our previous work [14] consists of the following: First, the transition from a set of time intervals $I_{i}$ to the points $\omega\left(T_{i}\right)$ $=2 \pi / I_{i}$, appropriate to the values of the average instanta- neous frequency during a return time $I_{i}=T_{i+1}-T_{i}$, is carried out; $T_{i}$ are the times of crossing of a threshold level (i.e., the times when spikes occur). Second, the points $\omega\left(T_{i}\right)$ are interpolated by a smooth function (also a cubic spline) $\omega_{\text {int }}(t)$ for transformation into a signal with constant time step [Fig. 2(b)], used for reconstruction of the attractor. It was shown [14] that the obtained temporal dependence allows us to describe the qualitative behavior of the average instantaneous frequency [Fig. 2(b)], and that the reconstructed attractor maintains the properties of the chaotic oscillations $S(t)$ [Fig. 3(c)].

Although in the present case we may consider other techniques for computing dynamical characteristics (see, for example, Refs. [28,32]), we decided to use the same approach and to estimate $\lambda_{1}$ from $\omega_{\text {int }}(t)$ by means of the method of Wolf et al. [26]. We suppose that the interpolation allows us to decrease orientational errors in the same way as for IF ISI's.

Some explanations to the method suggested by Janson et al. [14] may be appropriate. The discussed technique does not apply to the analysis of periodic oscillations of a period 1. In this case $\omega\left(T_{i}\right)=$ const, and we have a single point in a phase space. In the presence of noise we obtain some distribution of return times, but an estimation of the dynamical properties does not allow us to obtain true results. For complex periodic or chaotic regimes a transition to slower temporal variations occurs [i.e., $\omega_{\text {int }}(t)$ is a slower temporal function in comparison with $S(t)$ ]. However, the metrical and dynamical characteristics are maintained. This is confirmed by a computation of the largest Lyapunov exponent for the Rössler system, the Anishchenko-Astakhov oscillator, and a series of other models [14].

To examine the workability of the technique for LCE estimation in the presence of noise, we added normally distributed random values to the forcing signal (with a variance of $1 \%$ of the amplitude) and to the threshold level (1\% of the value $\theta$ for IF ISI's, and $10^{-2}$ for TC ISI's). The obtained results [Figs. 3(b) and $3(\mathrm{~d})$ ] testify to the stability of the algorithm to weak disturbances.

Figures 3(c) and 3(d) correspond to a threshold $\Theta=0$, i.e., to the introduction of a Poincaré section $x=0$ of the DS. In Sec. IV we shall discuss how the choice of a threshold level influences the result of LCE computations from TC ISI's.

\section{INFLUENCE OF THE THRESHOLD LEVEL}

The problem that we consider in the present study may be reduced to the following: Can the dynamical characteristics of a chaotic attractor be estimated only from a sequence of return times to a secant plane. If yes (what, actually, follows from the results of Hegger and Kantz [10]), under what conditions? Special interest is connected with the case when the threshold level $\Theta$ is introduced in such a way that not all the loops of the phase trajectory cross the secant plane $S=\Theta$. Consideration of this case will allow us to formulate limitations to our abilities to estimate $\lambda_{1}$ from interspike intervals. To be sure in our conclusions, we shall compare the results obtained with three different DS's chosen as the source of chaotic oscillations $S(t)$, namely, the Rössler system, the nephron model [33], and the $\beta$-cell model $[3,36]$. We shall 

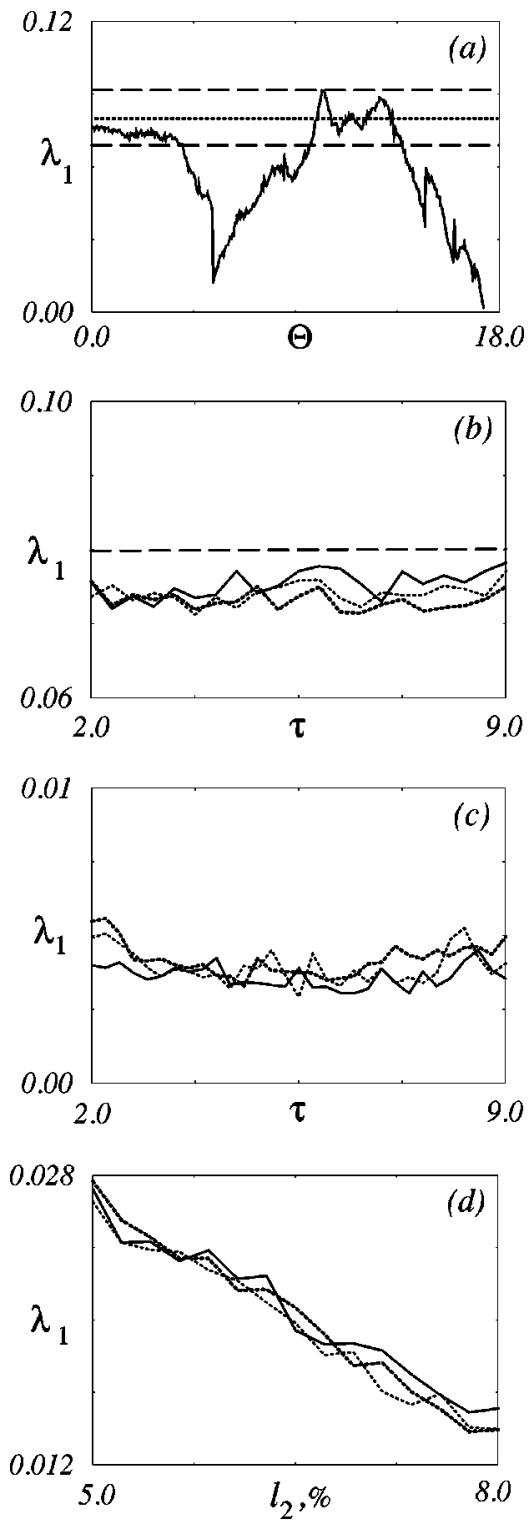

FIG. 4. (a) The largest LCE computed from TC ISI's vs threshold level $\Theta$. Dashed lines indicate the ranges of error $\pm 12 \%$. (b) and (c) The positive exponent vs $\tau$ at various $l_{2}$ 's for the thresholds $\Theta=11$ and 5.35. (d) The maximal LCE vs $l_{2}$ at various $\tau$ 's for the threshold level $\Theta=5.35$.

compare also the "variable evolution time" and the "fixed evolution time", algorithms [26] for the largest LCE estimation.

\section{A. Rössler system}

Consider Eqs. (6) and introduce, as illustrated in Fig. 1(b), a secant plane $x=\Theta$. Here, $S(t)=x(t)$. The largest Lyapunov exponent computed from TC ISI's at the different values of the threshold level using the "variable evolution time" algorithm [26] demonstrates a rather complex dependence on $\Theta$. This is shown in Fig. 4(a). As one can see from this figure there are two regions $(0 \leqslant \Theta<4$ and $10<\Theta$ $<13.5$ ), within the limits of which the dynamical properties of the chaotic attractor can be determined with good accuracy (the error of estimation for $\lambda_{1}$ does not exceed 10-
$15 \%$, and may be less if the length of time series increases). The dependence $\lambda_{1}(\Theta)$ in Fig. 4(a) was obtained for series of about 2000 return times.

Outside of the above intervals, the results differ significantly from the value $\lambda_{1}$ corresponding to the chaotic input signal. The maximal error of estimation occurs for $\Theta \approx 5.35$. More detailed calculations of $\lambda_{1}$ performed for several thresholds are shown in Figs. 3(c) $(\Theta=0), 4(b)(\Theta=11)$, $4(\mathrm{c})$, and $4(\mathrm{~d})(\Theta=5.35)$. The sensitivity of the algorithm to the choice of $l_{2}$ does not allow us to obtain an authentic estimation of the degree of chaoticity in the latter case.

Aiming to explain the complex behavior of $\lambda_{1}(\Theta)$, we have analyzed the structure of the return times (Fig. 5). In the region $0 \leqslant \Theta<4$, a crossing of the threshold level occurs during each oscillation. The sequence of time intervals (TC ISI's) is stationary [Fig. 5(a)], and the size of the attractor reconstructed from the interpolated temporal dependence $\omega_{\text {int }}(t)$ using the delay method is defined by the distribution of $I_{i}$ [Figs. 5(b) and 5(c)].

An increase of $\Theta$ leads to the absence of a threshold crossing during some oscillations: $I_{i}$ 's arise with large values [Fig. 5(d)]. The presence of several temporal scales in a sequence of TC ISI's leads to the appearance of several spatial scales in the reconstructed attractor [Fig. 5(e)], and because of different probabilities for large and small $I_{i}$ 's the phase trajectory will belong to the smaller region of a phase space ( $\approx 10-15 \%$ of the size of attractor) for a large part of the time [Fig. 5(e)]. The condition of linear approximation (parameter $l_{2}$ ) should now be introduced for each spatial scale separately; however, even at the essential reduction of the given parameter the dependence $\lambda_{1}\left(l_{2}\right)$ similar to Fig. $4(\mathrm{~d})$ is kept.

With further increase of $\Theta$, time intervals occur corresponding to three oscillations [Fig. 5(g)]. However, if the probabilities of various $I_{i}$ 's are of the same order the sequence of TC ISI's [Fig. 5(g)] preserves the dynamical properties of the forcing signal $S(t)$. In the region $\Theta>13.5$ there are time intervals among $I_{i}$ 's corresponding to five or more oscillations, with probabilities that are very small, but distinct from zero [Figs. 5(j) and 5(1)]. In this region it is obviously impossible to compute the value of the largest LCE.

On the basis of the above investigations, we can formulate a number of conditions under which one can estimate the dynamical characteristics of the Rössler system from TC ISI's using the "variable evolution time" algorithm [26].

(1) The sequence of $I_{i}$ 's must be stationary, and the probabilities of various return times in the distribution function must be approximately of the same magnitude (for ranges of $I_{i}$ 's with probabilities distinct from zero).

(2) The average value of the time interval $(\bar{I})$ should not exceed the characteristic temporal scale of the system under study (prediction time or Lyapunov time $[8,37]$ ).

Let us discuss these conditions. The first condition requires an approximate balance of times during which the phase trajectory belongs to various regions of the reconstructed chaotic attractor in the presence of several spatial scales. The second condition arises from the following problem: The increase of the average time interval $\bar{I}$ under a shift of the threshold level and the resulting excess of some characteristic temporal scale of the system (the prediction time) 

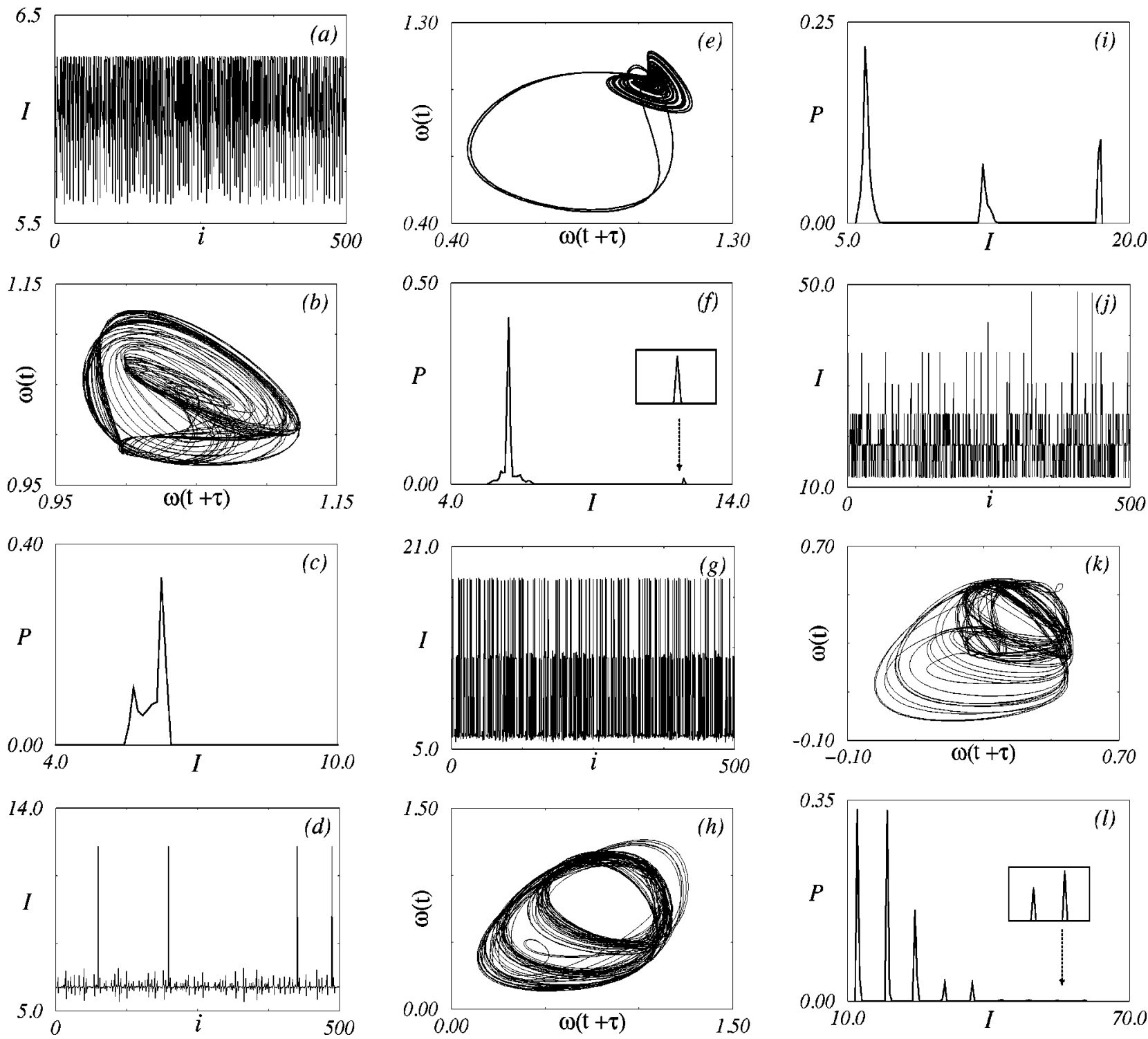

FIG. 5. The sequences of TC ISI's, phase portraits restored from the interpolated temporal dependences $\omega_{\text {int }}(t)$ (for simplicity we have dropped the designation "int" in the figures) and distribution functions appropriate to $\Theta=0[(\mathrm{a})-(\mathrm{c})], \Theta=5.35[(\mathrm{~d})-(\mathrm{f})], \Theta=11[(\mathrm{~g})-(\mathrm{i})]$, and $\Theta=15[(\mathrm{j})-(\mathrm{l})]$.

lead to a loss of information about the dynamics. As a consequence, the maximal value $\lambda_{1}$ which can be computed from the TC ISI series decreases inversely proportional to $\bar{I}$ [Fig. 6(a)]. However, even if the restrictions on $\bar{I}$ do not allow us to obtain the largest Lyapunov exponent the technique for LCE computation can serve as a qualitative estimation of a measure of chaoticity. For example, Fig. 6(b) shows the dependence of $\lambda_{1}$ on the control parameter $c$ of Eqs. (6) computed by the method of Refs. [21,22] in comparison with results of an estimation of the largest LCE from TC ISI's measured by fixation of the fifth crossing of a secant plane. We note the qualitative agreement in the variation of the two curves.

The above results were obtained within the framework of the "variable evolution time" algorithm which realizes the replacement procedures whenever a fixed spatial separation between nearby phase trajectories is reached (the condition of linear approach). As one can see, for the case $\Theta=5.35$ this technique may not work for attractors with several spa- tial scales in phase space. Formally, however, the algorithm of Ref. [26] has no limitations with respect to how the replacement procedures can be carried out.

In particular, it is possible to analyze the average rate of divergence for a fixed time span $T_{r}$ (the "fixed evolutiontime"' algorithm of Ref. [26]). However, in this case we have an additional parameter of the numerical computations. If $T_{r}$ is chosen small, the calculation time increases significantly (the search for nearest neighbors at each replacement procedure taking a major part of the time). Replacements that are performed too often may also lead to an increase of the orientational errors. On the other hand, having chosen a large $T_{r}$ we risk obtainning an underestimated value $\lambda_{1}$ if the divergence of nearby trajectories leaves the frameworks of the linear approach during the given time span. To obtain an authentic estimation of the largest LCE, its dependence from $T_{r}$ should be investigated, along with its dependence on the time delay, embedding dimension, etc.

The "fixed evolution time" algorithm [26] requires more calculation time. However, it allows us to estimate dynami- 

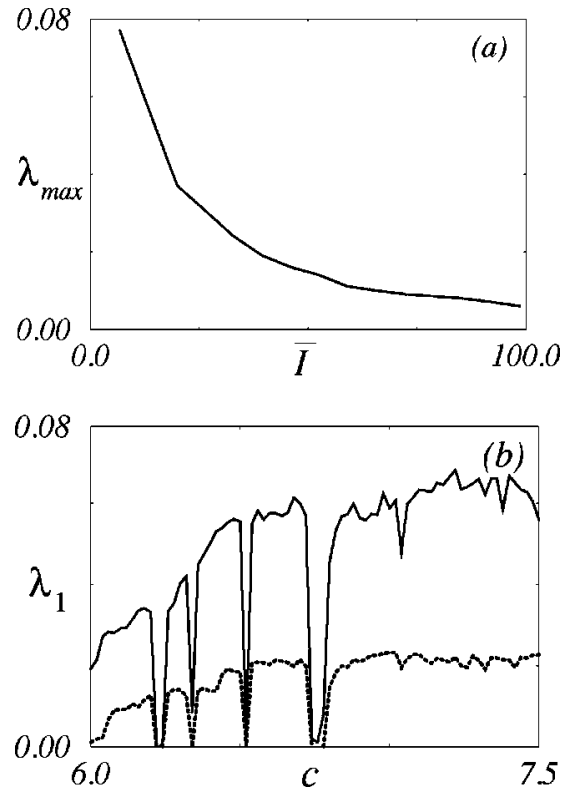

FIG. 6. (a) Estimation of the maximal LCE when $\bar{I}$ exceeds the characteristic temporal scale of a system. (b) The largest exponent computed using the method of Refs. $[21,22]$ vs a control parameter of the Rössler system (solid curve) and the values $\lambda_{1}$ estimated from TC ISI's measured from every fifth crossing of the secant plane (dashed curve).

cal characteristics of chaotic attractors at the presence of several clearly expressed temporal scales, when it is not always obvious how to introduce the condition of linear approach (parameter $l_{2}$ in our designations) (Fig. 7). Thus the first of the two conditions is no longer necessary.

So far, we have mainly discussed the technical aspects of the method of Ref. [26]. If, aiming to generalize the obtained
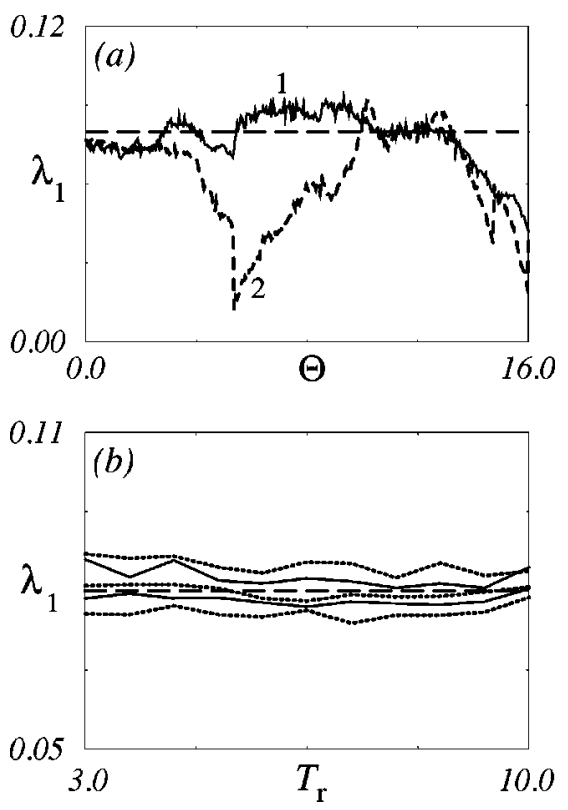

FIG. 7. (a) The largest LCE computed from TC ISI's vs threshold level $\Theta$ appropriate to the replacement procedures after a fixed time span (curve 1), and after a fixed spatial separation (curve 2). (b) The values of $\lambda_{1}$ vs $T_{r}$ at various $\tau \in[2 ; 5]$ for the threshold $\Theta=5.35$.
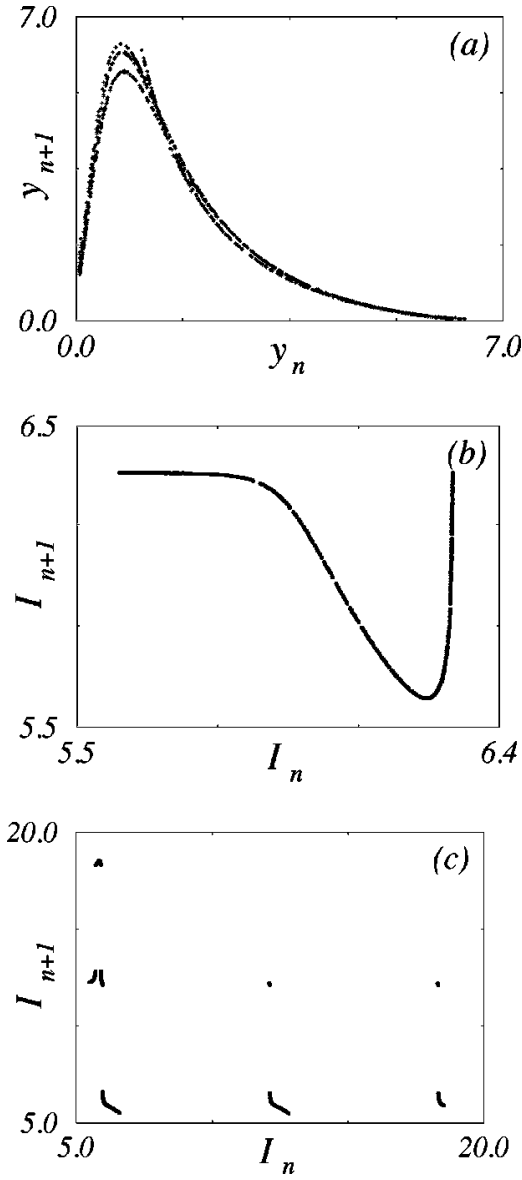

FIG. 8. (a) Poincaré map of the Rössler system. (b) and (c) Return time maps appropriate to $\Theta=0$ and 11 , respectively.

results, we neglect for a moment the details of the algorithm, then we can say that the sequence of return times maintains the dynamical properties of a chaotic attractor even in the case when not all the phase trajectories cross the secant plane. Thus, at the restriction to the average value of $I_{i}$ 's (second condition), the largest Lyapunov exponent is invariant to the choice of a threshold level.

This conclusion is not trivial. A shifting of the secant plane clearly results in essential changes of the structure of the return time map. While at the correct choice of the secant (when all the phase trajectories cross it) the return time map is similar to some extent to the Poincaré map [Figs. 8(a) and 8(b)], when shifting the threshold level the situation becomes complicated [Fig. 8(c)]. Before discussing the obtained results let us consider other sources of chaotic oscillations.

\section{B. Nephron model}

Using the Rössler system as an example, we have found that the largest LCE computed by the method of Ref. [26] does not depend significantly on the details of the replacement procedures if a sequence of TC ISI's contains only one temporal scale [Fig. 5(c)]. This is the simplest possible case. Aiming to test the invariance of dynamical characteristics to the change of a threshold, we are interested in processing interspike intervals with several scales (i.e., with larger ranges of $I_{i}$ 's). One system demonstrating such a behavior, in addition to the dependence on the choice of a threshold, level, is the nephron model [33]. 
The nephron is the functional unit of the kidney. Its main structure is described in the publications by Mosekilde and Barfred et al. [33]. Here a dynamical model of nephron autoregulation is presented in terms of the six ordinary differential equations

$$
\begin{gathered}
\frac{d P_{t}}{d t}=\frac{1}{C_{\text {tub }}}\left[\left(1-H_{a}\right)\left(1-\frac{C_{a}}{C_{e}}\right) \frac{P_{a}-P_{g}}{R_{a}}-F_{r e a b}-\frac{P_{t}-P_{d}}{R_{H e n}}\right], \\
\frac{d r}{d t}=v_{r}, \quad \frac{d v_{r}}{d t}=\frac{P_{a v}-P_{e q}}{\omega}-d v_{r}, \\
\frac{d X_{1}}{d t}=\frac{P_{t}-P_{d}}{R_{H e n}}-\frac{3 X_{1}}{T}, \quad \frac{d X_{2}}{d t}=\frac{3\left(X_{1}-X_{2}\right)}{T}, \\
\frac{d X_{3}}{d t}=\frac{3\left(X_{2}-X_{3}\right)}{T} .
\end{gathered}
$$

An explanation of the variables and parameter values is given in Appendix A. The description of Eqs. (8) in more detail can be found in Ref. [33] and references therein. We may note, however, that $P_{t}$ represents the fluid pressure in the proximal tubule immediately after the glomerulus. Measurements of this pressure for anesthetized rats show characteristic self-sustained oscillations with a period of 20-40 sec [34], and for rats with elevated blood pressure the oscillations tend to become chaotic [35]. $r$ denotes the radius of the afferent arteriole leading blood to the nephron, and the variables denoted $X_{1}, X_{2}$, and $X_{3}$ are intermediate variables in a third order smooth delay. In total, the model represents a feedback (the tubuloglomerular feedback) with a time delay $T$ and a relatively high loop gain. This gain is controlled to a large extent by the relation between the equilibrium pressure $P_{e q}$ in the active part of the afferent arteriole and the flow of nephronic liquid into the loop of Henle $F_{H e n}=\left(P_{t}\right.$ $\left.-P_{d}\right) / R_{H e n}$. The model represents a relatively accurate account of the basic physiological mechanisms responsible for the chaotic dynamics, and over the years it has been tested and examined in many different ways.

Figures 9(a) and 9(b) show the two-dimensional projection of the chaotic attractor being the solution to the nephron model and the temporal dependence of the first variable, the tubular pressure $P_{t}$. For any $\Theta$ [Fig. 9(b)] the sequence of return times to a secant plane [Fig. 9(c)] is similar to the TC ISI series of a Rössler system at large threshold levels. This means that the same problems arise, namely, attractors with several spatial scales, sensitivity to the choice of replacement procedure while computing $\lambda_{1}$, etc.

The results of an estimation of the largest LCE at different thresholds are given in Fig. 10(a). We can see that the "variable evolution time" algorithm [26], with the replacement procedures defined in terms of a fixed spatial separation between phase orbits, again lead to underestimated values when chaotic regimes with clearly expressed temporal scales are analyzed [curve 1 in Fig. 10(a)]. An acceptable accuracy is obtained only under the condition that the probabilities of various $I_{i}$ 's in the distribution function are of the same order $(1.2<\Theta<1.6)$. Computing $\lambda_{1}$ by means of the "fixed evolution time" algorithm [curve 2 in Fig. 10(a)] is more effective. Thus the invariance of the dynamical characteristics is
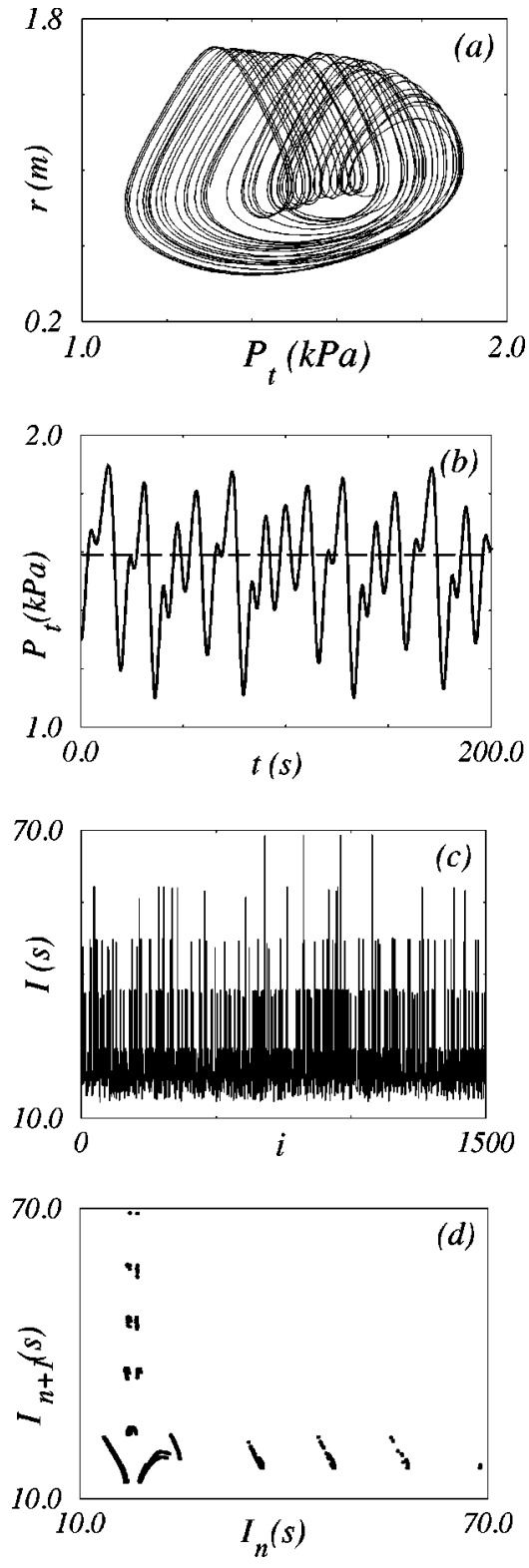

FIG. 9. (a) and (b) The solution of Eqs. (8) in a chaotic regime. (c) and (d) Series of TC ISI's and a return time map appropriate to $\Theta=1.3$.

confirmed at the restriction to $\bar{I}$. Similar results can be obtained if we compute $\lambda_{1}$ when changing the control parameter of Eqs. (8). This is illustrated in Fig. 10(b) (also see Appendix A).

\section{Pancreatic $\boldsymbol{\beta}$-cell model}

A very different example of a system with several clearly expressed temporal scales is a burst oscillator (see Ref. [3], and references therein). Bursting is a slow alternation between a silent phase and an active phase of fast oscillations.

Consider the equations of the $\beta$-cell model proposed by Sherman [3]:

$$
\begin{gathered}
\frac{d V}{d t}=\left[-I_{C a}-I_{K}-g_{S} S\left(V-V_{K}\right)\right] / \tau, \\
\frac{d n}{d t}=\lambda\left(n_{\infty}-n\right) / \tau, \quad \frac{d S}{d t}=\left(S_{\infty}-S\right) / \tau_{S},
\end{gathered}
$$



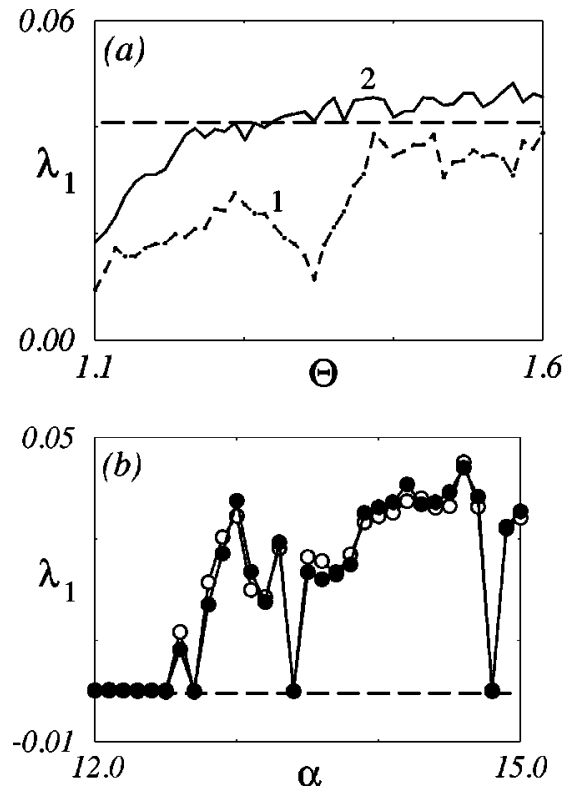

FIG. 10. (a) The largest LCE computed from TC ISI's vs threshold level $\Theta$ appropriate to the replacement procedures after a fixed spatial separation (curve 1), and after a fixed time span (curve 2). The dashed line indicates the value of $\lambda_{1}$ estimated from $P_{t}(t)$ using the method of Ref. [26]. (b) The dependence of $\lambda_{1}$ on the control parameter of Eqs. (8) (see Appendix A) computed from $P_{t}(t)$ using the algorithm of Ref. [26] (black points) and from TC ISI's (white points).

$$
\begin{gathered}
I_{C a}(V)=g_{C a} m_{\infty}\left(V-V_{C a}\right), \quad I_{K}(V, n)=g_{K} n\left(V-V_{K}\right), \\
x_{\infty}=\frac{1}{1+\exp \left[\left(V_{x}-V\right) / \theta_{x}\right]}, \quad x=m, n, S .
\end{gathered}
$$

In accordance with Ref. [36], $V$ is the voltage across the cell membrane, $n$ is the fraction of potassium channels that are open, and $S$ is a slow variable which may be related as the intracellular calcium concentration. Parameter values for system (9) are given in Appendix B.

The solution to Eqs. (9) is shown in Fig. 11(a). Shifting of a threshold level over wide ranges does not significantly change the probability distribution of TC ISI's [Fig. 11(b)] or the return time map [Fig. 11(c)]. Hence the largest Lyapunov exponent does not depend on the choice of the threshold, and can be estimated with good accuracy [Fig. 11(d)]. This again confirms the possibility of extracting dynamical properties of a chaotic attractor from a discrete sequence of time intervals in the presence of several temporal scales.

Within the scope of the present study we cannot suggest a more general proof of the invariance of the dynamical characteristics to the choice of a secant plane (assuming, of course, that the second condition is satisfied). A possible qualitative explanation consists of the following.

Consider, for simplicity, values of the average instantaneous frequency $\omega\left(t_{i}\right)=2 \pi / I_{i}$ at the moments $t_{i}=i \bar{I}$, i.e., with a uniform step in time. Let us choose two pieces of the signal $S(t)$, each containing five oscillations as shown in Fig. 12(a). For each of these pieces a small shift of the threshold level leads to the situation when, during the third oscillation, the trajectory does not cross the threshold any longer [Fig.
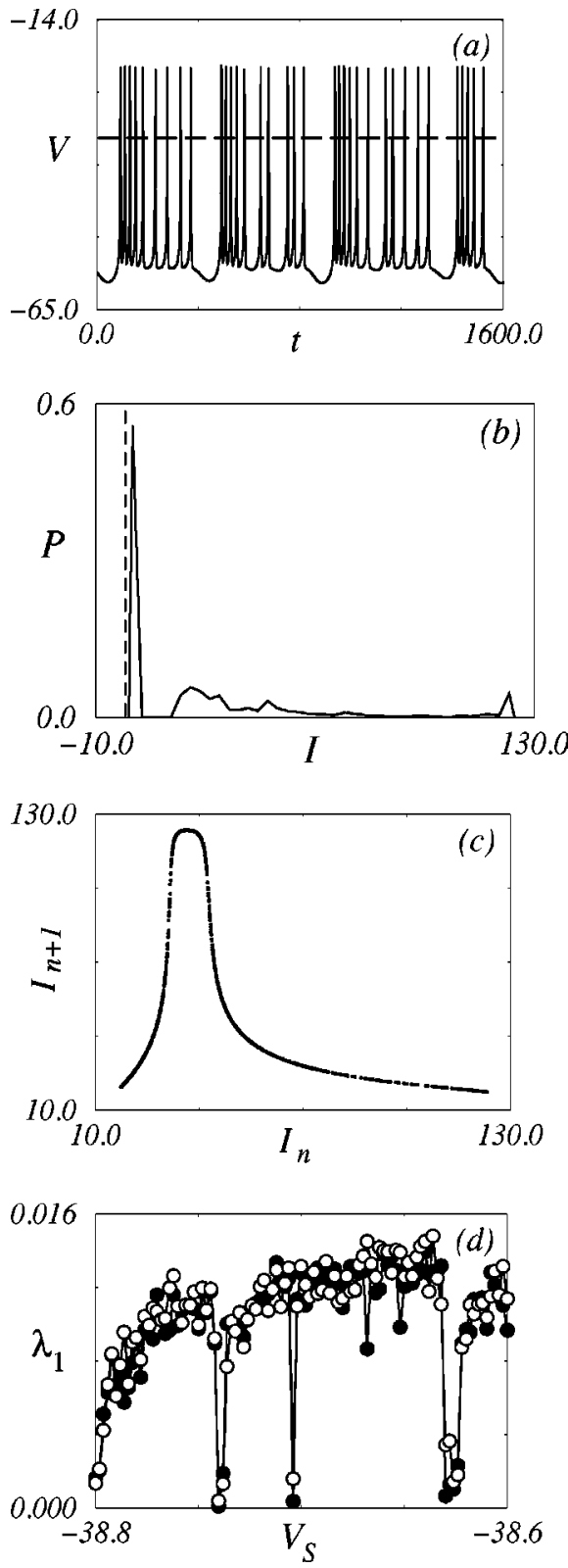

FIG. 11. (a) The potential of the cell membrane. (b) The probability distribution of return times. (c) Return time map. (d) The largest LCE vs the control parameter computed from $V(t)$ by means of the method of Refs. [21,22] (black points), and directly from return times (white points).

12(a)]. At the transition to interpolated temporal dependences, for the level $\Theta_{1}$ we shall obtain two smooth curves $\omega^{1}(t)$ and $\omega^{2}(t)$ passing through the points $\omega_{1}^{1}=\omega_{\text {int }}\left(t_{i}\right)$ $=2 \pi / I_{i}, \ldots, \omega_{4}^{1}=\omega_{i n t}\left(t_{i+3}\right)=2 \pi / I_{i+3}$ and $\omega_{1}^{2}=\omega_{\text {int }}\left(t_{j}\right)$ $=2 \pi / I_{j}, \ldots, \omega_{4}^{2}=\omega_{i n t}\left(t_{j+3}\right)=2 \pi / I_{j+3}$ at the moments $t_{i}, \ldots, t_{i+3}$ and $t_{j}, \ldots, t_{j+3}$ [Fig. 12(b)]. We indicate each of two chosen pieces of the signal $S(t)$ by indexes $i$ and $j$. The $t_{i}$ 's and $t_{j}$ 's refer to the times when the given pieces cross the threshold $\Theta_{1}$. If the value $D_{0}=\left|\omega_{1}^{1}-\omega_{1}^{2}\right|$ is small enough, the condition of linear approach is also satisfied for the distance between the curves during the computation time (three interspike intervals); then the one-dimensional analogue of the local Lyapunov exponent can be determined as follows [Fig. 12(b)]: 

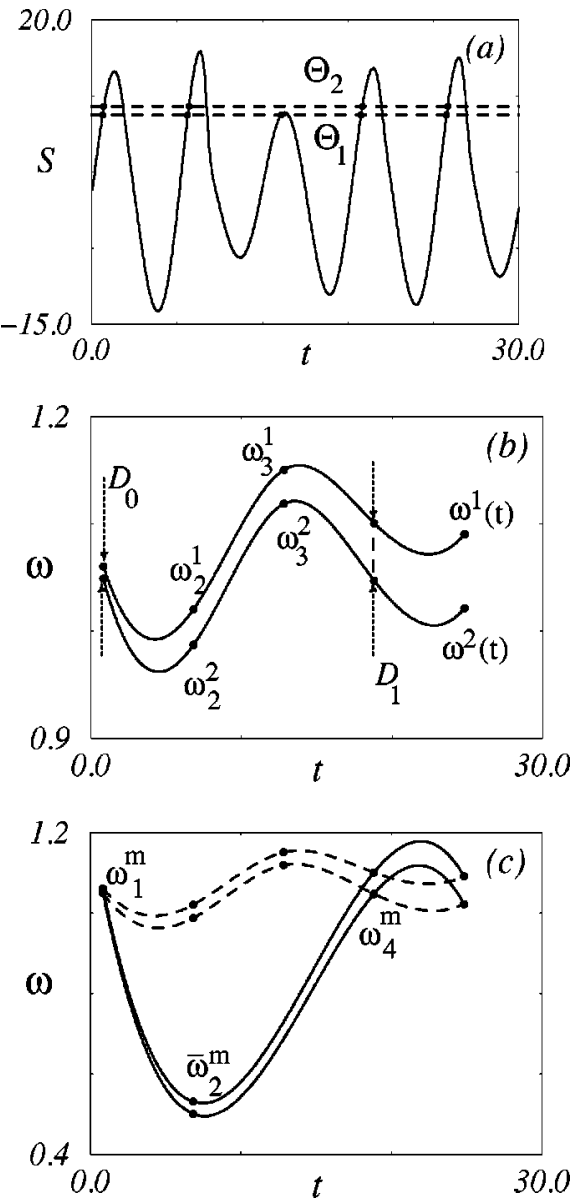

FIG. 12. (a) A piece of the signal $S(t)$ and two thresholds $\Theta_{1}$ $=9$ and $\Theta_{2}=10$. (b) The interpolated temporal dependences $\omega^{1}(t)$ and $\omega^{2}(t)$ for the two pieces of $S(t)$ appropriate to the threshold $\Theta_{1}$. Black points indicate the values of $\omega_{k}^{m}$. (c) The interpolated temporal dependences $\omega^{1}(t)$ and $\omega^{2}(t)$ for two pieces of $S(t)$ appropriate to the threshold $\Theta_{2}$. Dashed curves indicate the dependences shown in Fig. 12(b).

$$
\lambda_{1}\left(t_{0}\right)=\frac{1}{3 \bar{I}} \ln \frac{D_{1}}{D_{0}}, \quad D_{1}=\left|\omega_{4}^{1}-\omega_{4}^{2}\right|
$$

The insignificant shift of the threshold (the level $\Theta_{2}$ ) results in a change of the sequences $\omega_{k}^{m}$. For each of them the points $\omega_{1}^{m}$ and $\omega_{4}^{m}$ remain practically unchanged, and instead of $\omega_{2}^{m}$ and $\omega_{3}^{m}$ there will be one point $\overline{\omega_{2}^{m}}$, but with a value half as large as in the previous case [Fig. 12(c)]. This, in turn, results in changes of the rate of divergence of the trajectories at the local regions of the attractor. However, the average characteristics of the degree of chaoticity will remain the same as determined by Eq. (10) [Fig. 12(c)]. A similar discussion can be carried out for a nonuniform step in time. Clearly, these arguments do not serve as a strict mathematical proof, and should be interpreted only as a qualitative explanation.

Note that there are no theoretical results showing how an attractor's characteristics depend on the threshold level. In theory, the correct Lyapunov exponent can be found for any $\Theta$. For example, Sauer's theorem [7] has no limitations on the value of a threshold. But, in the work [6] (as well as in

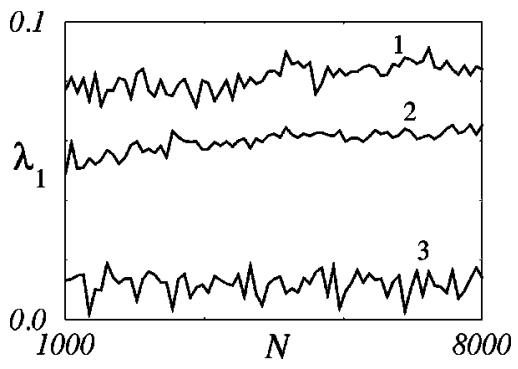

FIG. 13. The positive exponent estimated from the TC ISI series of the Rössler system vs the length of time series at various thresholds $\Theta=11$ (dependence 1), $\Theta=15$ (dependence 2), and $\Theta=17$ (dependence 3).

other papers, see e.g., Ref. [8]) it was mentioned that in practice (when processing finite amount of data), the quality of reconstruction depends on $\Theta$. Following Ref. [6], at some levels, "for a fixed embedding dimension the length of dynamical time spanned by a reconstructed ISI vector becomes comparable with the decorrelation time of the chaotic system, which has the effect of attractor degrading."

The aim of our paper is a numerical testing of the possibilities to estimate perhaps the most informative invariant of a complicated dynamical process (the largest LCE) from time intervals. Using several models we compute the LCE with good enough accuracy even in the case when TC ISI series contain large intervals provided that the mean return time does not exceed some temporal scale (which corresponds approximately to the Lyapunov time, i.e., to the inverse Lyapunov exponent). Moreover, if some loops of phase space trajectory fail to cross the threshold, the value of the LCE can be extracted almost with the same accuracy as in the case when the crossing of a threshold takes place during each oscillation. These results differ from the previous computing of correlation dimension [6], that was significantly more sensitive to the choice of $\Theta$. Similar results were obtained for other models [38].

Note also that, in practice (when processing finite amount of data), the results of numerical computations do not significantly depend on the length of time series if all other parameters are fixed (see Fig. 13, for example). The algorithm of Ref. [26] is considerably more sensitive to the choice of initial separations between two orbits in the phase space. This dependence is similar to the one in Fig. 4(d) for large thresholds, and gives no possibility of estimating the measure of chaoticity. The reason for this is as follows: When large ISI's are analyzed, a small (but finite) perturbation leaves the framework of the linear approach during the time between spikes, and can reach the size of attractor. As a result, an underestimated value of the LCE is obtained [see Fig. 4(a) for large thresholds].

\section{CONCLUSIONS}

In the present work we have considered two different models of spike generation (namely, the integrate-and-fire model and the threshold-crossing model), and have suggested a procedure for a transition to a continuous-time variation for IF ISI's, allowing us to reproduce qualitatively the linear transformation of an input signal for the given model. We have analyzed how the choice of threshold level influences the results of reconstruction of the dynamical 
characteristics from a series of return times (TC ISI's), and showed that under quite general conditions the largest Lyapunov exponent is invariant to the choice of a threshold, and can be determined from TC ISI's even in the case when not all loops of the phase trajectory cross the secant plane. Of course, when speaking about the invariance of dynamical properties we understand that the largest LCE can be estimated only with a certain accuracy, taking into account both the finite amount of data and the dependence of the algorithm [26] on parameters of the numerical computations. Let us note that, instead of shifting a secant plane, we could fix a constant value of a threshold $(\Theta>0)$ and change only the amplitude of the input signal $S(t)$. That would lead to similar conclusions.

In our paper the results obtained for a Rössler system, a nephron model, and a pancreatic $\beta$-cell model are demonstrated. Actually our study was not limited only to these systems. Similar conclusions follow from the analysis of other models of nonlinear dynamics and mathematical biology, for which the possibility of extracting dynamics is confirmed if the average value of the return times does not exceed a characteristic temporal scale of the dynamical system. A number of interesting results have been obtained for chaotic attractors with several equilibrium points relative to which the movement of a phase trajectory takes place (for example, the Lorenz system). However, the analysis of such systems is a rather complex problem, which will constitute a separate investigation.

\section{ACKNOWLEDGMENT}

This work was partly supported by INTAS Grant Nos. INTAS 96-0305 and YSF 99-4050.

\section{APPENDIX A: NEPHRON MODEL}

The relations between variables in Eqs. (8) are as follows:

$$
\begin{gathered}
R_{a}=R_{a, 0}\left[\beta+(1-\beta) r^{-4}\right], \\
P_{g}=P_{t}+a C_{e}+b C_{e}^{2}, \\
P_{a v}=0.5\left(P_{a}+P_{g}-\beta\left(P_{a}-P_{g}\right) \frac{R_{a, 0}}{R_{a}}\right), \\
P_{e q}=p_{1}(r-1)+p_{2} \exp ^{10(r-0.8)} \\
+\Psi\left(p_{3}+p_{4} r+\frac{p_{5}}{1+\exp ^{-13(r-0.4)}}\right), \\
\Psi\left(\frac{3 X_{3}}{T}\right)=\Psi_{\text {max }}-\frac{\Psi_{\text {max }}-\Psi_{\text {min }}}{1+\exp _{\alpha}\left(\frac{3 X_{3}}{T F_{H e n, 0}}-S\right)}, \\
S=1-\frac{1}{\alpha} \ln \left(\frac{\Psi_{e q}-\Psi_{\text {min }}}{\Psi_{\text {max }}-\Psi_{e q}}\right)
\end{gathered}
$$

The value of $C_{e}$ is uniquely defined from an algebraic equation:

$$
\begin{aligned}
(b+ & \left.\frac{R_{e}}{R_{a}} b H_{a}\right) C_{e}^{3}+\left(a+\frac{R_{e}}{R_{a}} b C_{a}\left(1-H_{a}\right)+\frac{R_{e}}{R_{a}} a H_{a}\right) C_{e}^{2} \\
& +\left(P_{t}-P_{v}+\frac{R_{e}}{R_{a}} a C_{a}\left(1-H_{a}\right)+\frac{R_{e}}{R_{a}}\left(P_{t}-P_{a}\right) H_{a}\right) C_{e} \\
& +\left(P_{t}-P_{a}\right) \frac{R_{e}}{R_{a}} C_{a}\left(1-H_{a}\right)=0 .
\end{aligned}
$$

Model variables.

\begin{tabular}{ll}
\hline \multicolumn{1}{c}{ Variable } & \multicolumn{1}{c}{ Explanation } \\
\hline$P_{t}$ & $\begin{array}{l}\text { Proximal tubular pressure } \\
\text { Radius of the active part of the afferent } \\
\text { arteriole }\end{array}$ \\
$X_{1}, X_{2}, X_{3}$ & $\begin{array}{l}\text { Intermediate variables in the delay chain } \\
C_{e}\end{array}$ \\
$P_{g}$ & $\begin{array}{l}\text { Plasma protein concentration in the effer- } \\
\text { ent arteriole }\end{array}$ \\
$R_{a}$ & $\begin{array}{l}\text { Glomerular pressure } \\
P_{e q}\end{array}$ \\
$P_{a v}$ & $\begin{array}{l}\text { Flow resistance of afferent arteriole } \\
\text { Equilibrium pressure in the variable part of } \\
\text { the afferent arteriole }\end{array}$ \\
& $\begin{array}{l}\text { Average pressure in the variable part of the } \\
\text { afferent arteriole }\end{array}$ \\
\hline
\end{tabular}

Model parameters.

\begin{tabular}{ll}
\hline$C_{\text {tub }}=3.0 \mathrm{~nL} / \mathrm{kPa}$ & $\Psi_{\text {min }}=0.20$ \\
$H_{a}=0.5$ & $\Psi_{\text {max }}=0.44$ \\
$P_{a}=13.3 \mathrm{kPa}$ & $\Psi_{\text {eq }}=0.38$ \\
$P_{v}=1.3 \mathrm{kPa}$ & $C_{a}=54 \mathrm{~g} / \mathrm{L}$ \\
$P_{d}=0.6 \mathrm{kPa}$ & $a=22 \times 10^{-3} \mathrm{kPa}(\mathrm{L} / \mathrm{g})$ \\
$F_{\text {hen }, 0}=0.2 \mathrm{~nL} / \mathrm{s}$ & $b=0.39 \times 10^{-3} \mathrm{kPa}(\mathrm{L} / \mathrm{g})^{2}$ \\
$F_{\text {reab }}=0.3 \mathrm{~nL} / \mathrm{s}$ & $p_{1}=1.6 \mathrm{kPa}$ \\
$R_{\text {hen }}=5.3 \mathrm{kPa}(\mathrm{s} / \mathrm{nL})$ & $p_{2}=6 \times 10^{-3} \mathrm{kPa}$ \\
$R_{a, 0}=2.3 \mathrm{kPa}(\mathrm{s} / \mathrm{nL})$ & $p_{3}=6.3 \mathrm{kPa}$ \\
$R_{e}=1.9 \mathrm{kPa}(\mathrm{s} / \mathrm{nL})$ & $p_{4}=7.2 \mathrm{kPa}$ \\
$\omega=20 \mathrm{kPa} \mathrm{s}$ & $p_{5}=4.7 \mathrm{kPa}$ \\
$d=0.04 \mathrm{~s}-1$ & $T=3 \mathrm{~s}$ \\
$\beta=0.67$ & $\alpha=14.5$
\end{tabular}

\section{APPENDIX B: PANCREATIC $\beta$-CELL MODEL}

Model parameters.

\begin{tabular}{ll}
\hline$g_{C a}=3.6$ & $V_{m}=-20 \mathrm{mV}$ \\
$g_{K}=10.0$ & $V_{n}=-16 \mathrm{mV}$ \\
$g_{S}=4.0$ & $V_{S}=-40 \mathrm{mV}$ \\
$\tau=20 \mathrm{~ms}$ & $\theta_{m}=12 \mathrm{mV}$ \\
$\tau_{S}=35 \mathrm{~s}$ & $\theta_{n}=5.6 \mathrm{mV}$ \\
$V_{C a}=25 \mathrm{mV}$ & $\theta_{S}=10 \mathrm{mV}$ \\
$V_{K}=-75 \mathrm{mV}$ & $\lambda=0.85$ \\
\hline
\end{tabular}


[1] H. C. Tuckwell, Introduction to Theoretical Neurobiology (Cambridge University Press, Cambridge, England, 1988), Vols. 1 and 2.

[2] Task Force of the European Society of Cardiology and the North American Society of Pacing and Electrophysiology, Circulation 93, 1043 (1996).

[3] A. Sherman, Bull. Math. Biol. 56, 811 (1994).

[4] A. Longtin, A. Bulsara, and F. Moss, Phys. Rev. Lett. 67, 656 (1991); A. Garfinkel, M.L. Spano, W.L. Ditto, and J.W. Weiss, Science 257, 1230 (1992); S.J. Schiff, K. Jerger, D.H. Duong, T. Chang, M.L. Spano, and W.L. Ditto, Nature (London) 370, 615 (1994); F. Moss and X. Pei, ibid. 376, 211 (1995); K.A. Richardson, T.T. Imhoff, P. Grigg, and J.J. Collins, Phys. Rev. Lett. 80, 2485 (1998); D. Christini and J.J. Collins, ibid. 75, 2782 (1995); X. Pei and F. Moss, Nature (London) 379, 618 (1996); P. So, E. Ott, S. Schiff, D. Kaplan, T. Sauer, and C. Grebogi, Phys. Rev. Lett. 76, 4705 (1996).

[5] T. Sauer, Phys. Rev. Lett. 72, 3811 (1994).

[6] R. Castro and T. Sauer, Phys. Rev. E 55, 287 (1997).

[7] T. Sauer, in Nonlinear Dynamics and Time Series, edited by C. Culter and D. Kaplan, Fields Institute Communications Vol. 11 (American Mathematical Society, Providence, RI, 1997), p. 63.

[8] D.M. Racicot and A. Longtin, Physica D 104, 184 (1997).

[9] W. Bialek, F. Rieke, R.R. de Ruyter van Steveninck, and D. Warland, Science 252, 1854 (1991); F. Gabbiani and C. Koch, Neural Comput. 8, 44 (1996).

[10] R. Hegger and H. Kantz, Europhys. Lett. 38, 267 (1997).

[11] R. Castro and T. Sauer, Phys. Rev. Lett. 79, 1030 (1997); Phys. Rev. E 59, 2911 (1999).

[12] M. Ding and W. Yang, Phys. Rev. E 55, 2397 (1997).

[13] F. Takens, in Dynamical Systems and Turbulence, edited by D. A. Rang and L. S. Young, Lecture Notes in Mathematics Vol. 898 (Springer-Verlag, Berlin, 1981), p. 366; N.H. Packard, J.P. Crutchfield, J.D. Farmer, and R.S. Shaw, Phys. Rev. Lett. 45, 712 (1980); T. Sauer, J.A. Yorke, and M. Casdagli, J. Stat. Phys. 65, 579 (1991); J.L. Breeden and N.H. Packard, Int. J. Bifurcation Chaos Appl. Sci. Eng. 4, 311 (1994).

[14] N.B. Janson, A.N. Pavlov, A.B. Neiman, and V.S. Anishchenko, Phys. Rev. E 58, R4 (1998).

[15] T. Sauer, J.A. Tempkin, and J.A. Yorke, Phys. Rev. Lett. 81, 4341 (1998).

[16] S.P. Dawson, Phys. Rev. Lett. 76, 4348 (1996).

[17] J.-M. Gambaudo and C. Tresser, Phys. Lett. A 94, 412 (1983); G. Froyland, K. Judd, and A.I. Mees, Phys. Rev. E 51, 2844 (1995).

[18] V.S. Anishchenko and M.A. Safonova, Pis'ma Zh. Tekh. Fiz. 12, 740 (1986) [Sov. Tech. Phys. Lett. 12, 305 (1986)]; V.S. Anishchenko and H.P. Herzel, ZAMP 68, 317 (1988).

[19] J.C. Sommer and E. Ott, Nature (London) 365, 136 (1993).

[20] B.R. Hunt and E. Ott, Phys. Rev. Lett. 76, 2254 (1996).
[21] G. Benettin, L. Galgani, A. Giorgilli, and J.M. Strelcyn, Meccanica 15, 9 (1980)

[22] I. Shimada and T. Nagashima, Prog. Theor. Phys. 61, 1605 (1979).

[23] W.E. Wiesel, Phys. Rev. E 47, 3686 (1993).

[24] W.E. Wiesel, Phys. Rev. A 46, 7480 (1992); J. Theiler and L.A. Smith, Phys. Rev. E 51, 3738 (1995); S. Dawson, C. Grebogi, T. Sauer, and J.A. Yorke, Phys. Rev. Lett. 73, 1927 (1994); G. Rangarajan, S. Habib, and R.D. Ryne, Phys. Rev. Lett. 80, 3747 (1998).

[25] S.V. Ershov and A.B. Potapov, Physica D 118, 167 (1998).

[26] A. Wolf, J.B. Swift, H.L. Swinney, and J.A. Vastano, Physica D 16, 285 (1985).

[27] J.P. Eckmann, S.O. Kamphorst, D. Ruelle, and D. Cilberto, Phys. Rev. A 34, 4971 (1986); M. Sano and Y. Sawada, Phys. Rev. Lett. 55, 1082 (1985); R. Brown, Phys. Rev. E 47, 3962 (1993); U. Parlitz, Int. J. Bifurcation Chaos Appl. Sci. Eng. 2, 155 (1992).

[28] H. Kantz, Phys. Lett. A 185, 77 (1994).

[29] J.P. Eckmann and D. Ruelle, Physica D 56, 185 (1992); R. Brown, P. Bryant, and H.D.I. Abarbanel, Phys. Rev. A 43, 2787 (1991); A. Karantonis and M. Pagitsas, Phys. Rev. E 53, 5428 (1996); X. Zeng, R. Eykholt, and R.A. Pielke, Phys. Rev. Lett. 66, 3229 (1991); P. Bryant, R. Brown, and H.D.I. Abarbanel, Phys. Rev. Lett. 65, 1523 (1990).

[30] A. Potapov, Physica D 101, 207 (1997).

[31] G. Paladin, M. Serva, and A. Vulpiani, Phys. Rev. Lett. 74, 66 (1995); V. Loreto, G. Paladin, and A. Vulpiani, Phys. Rev. E 53, 2087 (1996).

[32] P. Faure and H. Korn, Physica D 122, 265 (1998).

[33] E. Mosekilde, Topics in Nonlinear Dynamics. Applications to Physics, Biology and Economic Systems. (World Scientific, Singapore, 1996), p. 380; M. Barfred, E. Mosekilde, and N.-H. Holstein-Rathlou, Chaos 6, 280 (1996); M. D. Andersen and N. Carlsson, M.S. thesis, The Technical University of Denmark, 1999

[34] P.P. Liyssac and L. Banmbach, Acta Physiol. Scand. 117, 415 (1993).

[35] K.-P. Yip, N.-H. Holstein-Rathlou, and D.J. Marsh, Am. J. Phys. 261, F400 (1991).

[36] D. Mears, N.F. Sheppard, I. Atwater, E. Rojas, R. Bertram, and A. Sherman, J. Membr. Biol. 155, 47 (1997).

[37] J.D. Farmer and J.J. Sidorowich, Phys. Rev. Lett. 59, 845 (1987); J. Brindley and T. Kapitaniak, in: Chaotic, Fractal and Nonlinear Signal Processing, edited by R. A. Katz, AIP Conf. Proc No. 375, (AIP, New York, 1996), p. 605.

[38] A.N. Pavlov, E. Mosekilde, and V. S. Anishchenko, in Stochaos: Stochastic and Chaotic Dynamics in the Lakes, edited by D.S. Broomhead, E.A. Luchinskaya, P.V.E. McClintock, and T. Mullin, AIP Conf. Proc. No. 502 (AIP, New York, 1999), p. 611. 